\title{
INFLUÊNCIA DA FIBRA DE CARBONO EM NÃOTECIDOS DE ISOLAMENTOS
}

\author{
Yuri Fazion Gradela ${ }^{1}$, Ana Luiza De Rosa ${ }^{1}$, Marcia Zanini Carrieri ${ }^{1}$ e Camilla Borelli ${ }^{1}$ \\ ${ }^{1}$ Centro Universitário da FEI - Fundação Educacional Inaciana "Pe. Sabóia de Medeiros" \\ E-mails: yuri.gradela@terra.com.br, analuiza.derosa@hotmail.com, \\ marcia.carriei@gmail.com, cborelli@fei.edu.br.
}

\section{RESUMO}

A crescente utilização de materiais têxteis no interior dos veículos automotivos empregados na absorção de ruídos do motor, do ambiente, isolamento térmico externo, do motor, amortecimento ou funções conjuntas, exigem alto desempenho e durabilidade dos materiais.

O presente estudo tem como objetivo demonstrar a influência do uso de fibras de carbono na composição de nãotecidos juntamente com poliéster, agulhados e termofixados.

A fibra de carbono possui grande resistência térmica e alta resistência à tração podendo substituir os aditivos químicos utilizados atualmente.

As amostras de nãotecido enriquecidas foram preparadas em diferentes proporções de composição com a finalidade de comparar suas propriedades quanto ao retardo à chama e resistência à tração.

Os ensaios realizados têm como referência as seguintes normas: ASTM D1230 Inflamabilidade à $45^{\circ}$, D6413 Inflamabilidade vertical, MVSS 302 Inflamabilidade horizontal, NBR 13041 Tração de nãotecidos método Grab e Tira.

\begin{abstract}
The increasing use of textiles in the interior of automotive vehicles used in absorption of engine and environment noise, external and motor insulation, damping or joint functions, require high performance and durability of materials.
\end{abstract}

This study aims to demonstrate the influence of the use of carbon fibers in the composition of nonwoven together with polyester needled and termofixed.

Carbon fiber has great thermal resistance and high tensile strength and can replace chemical additives used today.

The samples of non-woven enriched in different composition proportions were prepared in order to compare their properties in retarding flame and tensile strength.

Tests done have as reference the following standards: ASTM D1230 Flammability to $45^{\circ}$, D6413 Vertical flammability, Horizontal flammability MVSS 302, NBR 13041 Traction nonwoven Grab and Strip method. 


\section{INTRODUÇÃO}

As misturas de fibras têxteis semelhantes em comprimento e título são utilizadas há muito tempo visando à união de comportamentos distintos em um único produto e melhorar suas propriedades.

Estudos anteriores de FLOYD e TAYLOR [1] já demonstravam as propriedades especiais das fibras de carbono, em misturas ou puras, quanto sua alta resistência térmica e alta resistência mecânica. Sua utilização principal esta diretamente relaciona a indústria aeroespacial e automobilística.

As fibras de carbono mais utilizadas nos dias de hoje são classificadas como fibras inorgânicas e obtidas a partir do processo de oxidação e carbonização das fibras acrílicas [2]. O acrílico por sua vez é produzido principalmente nos processos de fiações do tipo "a seco" ou "a úmido", onde o polímero necessita da ação de secagem ou solvente para a polimerização final [3].

Em paralelo as fibras sintéticas ganharam o mercado com seu preço e grande disponibilidade de configurações. A fibra de poliéster é largamente utilizada, devido suas capacidades de durabilidade e propriedades termoplásticas. Obtida pelo processo de fiação via extrusão [3], é utilizada em diversos materiais têxteis através de fibras fios e misturas.

Para unir as qualidades destes dois materiais em aplicações comuns existentes na indústria automobilística, estudamos a utilização da mistura de fibras de carbono cortadas, em configurações semelhantes às fibras de poliéster em misturas consolidadas pelo processo de agulhagem resultando em um nãotecidos.

Os nãotecidos são estruturas planas formadas por véus e mantas de fibras orientadas aleatoriamente ou direcionais, consolidadas por processo térmico, mecânico ou químico [4]. Utilizados como isolantes térmicos e acústicos no interior do automóvel e no compartimento do motor, são de grande importância na segurança e desempenho mecânico [5], [6].

Buscando melhorar o desempenho do material utilizado, este trabalho tem como objetivo testar a influência da adição de fibras de carbono junto ao poliéster em proporções diferentes formando nãotecidos, maximizando o efeito de retardo às chamas [7] sem a emissão e gases tóxicos provocados por produtos químicos [8] e preservando suas propriedades mecânicas e acústicas.

\section{OBJETIVO}

1.1 Produzir seis amostras de nãotecidos em diferentes proporções de mistura conforme tabela 1, fixando a gramatura e realizar os ensaios de tração e inflamabilidade. 


\begin{tabular}{|c|c|c|}
\hline Mistura 400 $\left(\mathbf{g} / \mathbf{m}^{\mathbf{2}}\right)$ & Poliéster (\% PES) & Carbono (\% C) \\
\hline 1 & 100 & 0 \\
\hline 2 & 80 & 20 \\
\hline 3 & 60 & 40 \\
\hline 4 & 40 & 60 \\
\hline 5 & 20 & 80 \\
\hline 6 & 0 & 100 \\
\hline
\end{tabular}

Tabela 1: Misturas iniciais intervalo $20 \%$.

1.2 Identificar o intervalo de maior alteração de comportamento e produzir três novas amostras de diferentes misturas, conforme tabela 2, fixando a gramatura e realizar novamente os ensaios.

\begin{tabular}{|c|c|c|}
\hline Mistura 400 $\left(\mathrm{g} / \mathbf{m}^{2}\right)$ & Poliéster (\% PES) & Carbono (\% C) \\
\hline 7 & $+/-15$ & $+/-5$ \\
\hline 8 & $+/-10$ & $+/-10$ \\
\hline 9 & $+/-5$ & $+/-15$ \\
\hline
\end{tabular}

Tabela 2: Misturas secundárias intervalo 5\%.

1.3 Demonstrar graficamente e visualmente os resultados obtidos.

\section{MATERIAIS E MÉTODOS}

\subsection{Seleção das fibras.}

Para este estudo foram utilizadas fibras de carbono e poliéster conforme as especificações da tabela 3.

\begin{tabular}{|c|c|c|}
\hline Material & Título (dTex) & Comprimento (mm) \\
\hline Poliéster & 8,7 & 65 \\
\hline Carbono & 2,8 & 60 \\
\hline
\end{tabular}

Tabela 3: Especificação de fibras.

\subsection{Preparação das misturas e amostras.}

Para cada mistura estabelecida foram pesadas e separadas quatro porções de $75 \mathrm{~g}$ para cada véu a ser cardado.

As porções de fibras foram cardadas duas vezes a fim de obter maior homogeneização de fibras. Cada par de véus foi disposto à $90^{\circ}$ em relação ao outro proporcionando fibras orientadas horizontalmente e verticalmente.

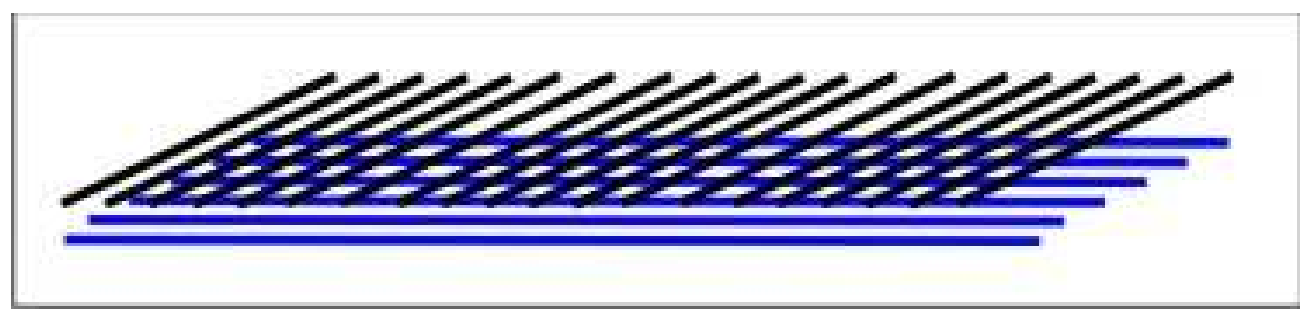

Figura 1: Posição dos véus. 
Os véus foram consolidados via agulhagem superior unidirecional, onde são perfurados por agulhas contendo ranhuras que levam as fibras superiores para o inferior dos véus entrelaçando as fibras [9].

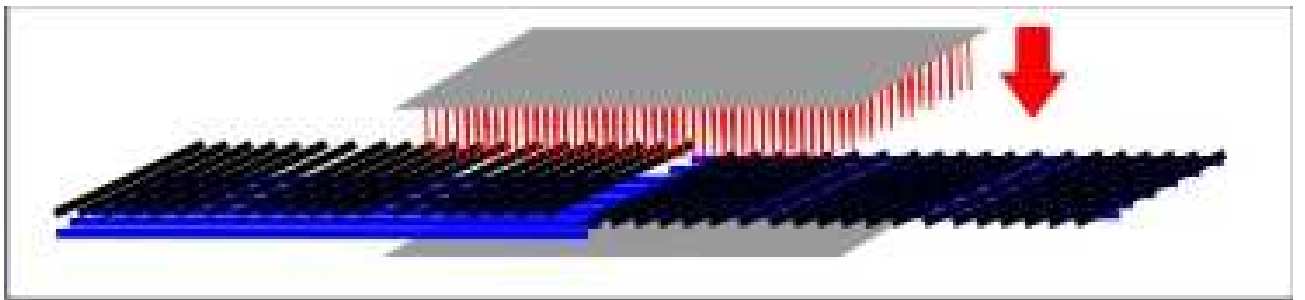

Figura 2: Esquema de agulhagem.

Após a preparação dos nãotecidos, foram cortadas amostras conforme especificação, para realização de ensaios.

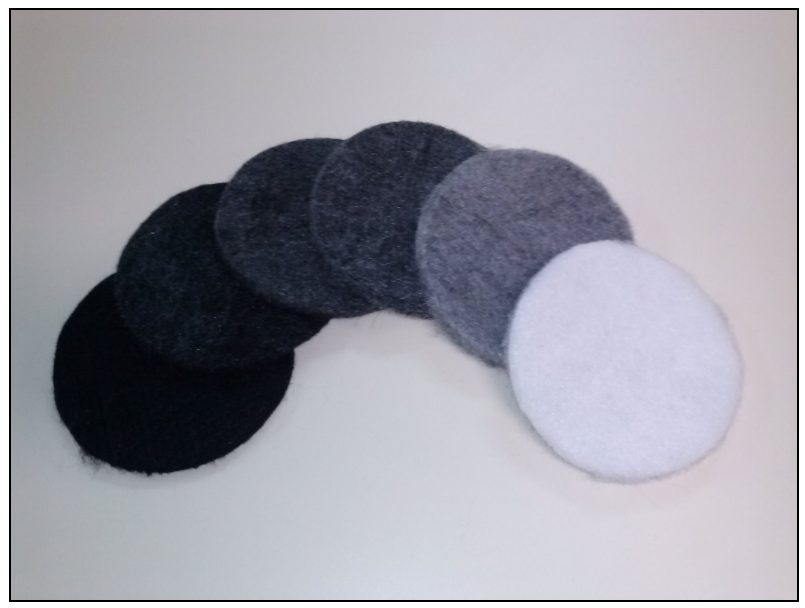

Figura 3: Amostras.

\subsection{Ensaios.}

Os ensaios de tração foram realizados em dinamômetro de coluna com deslocamento constante conforme norma NBR 13041. 


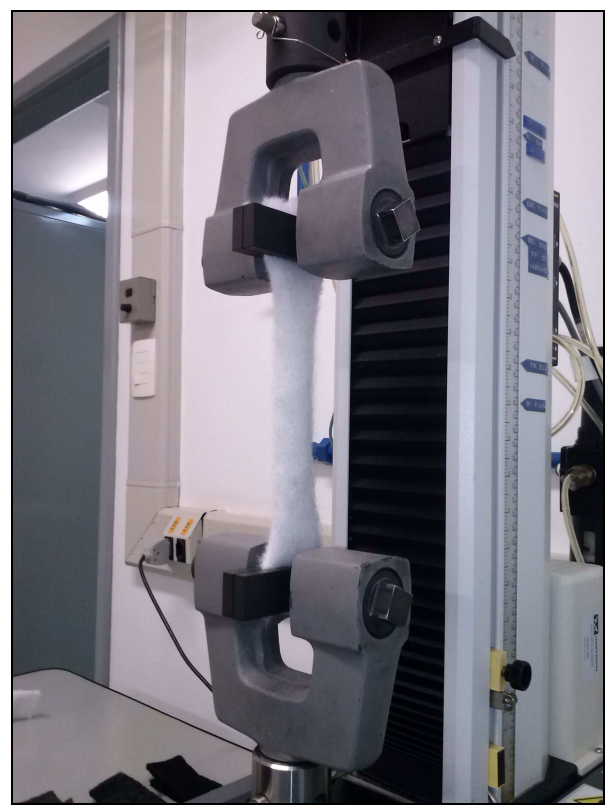

Figura 4: Ensaio de tração.

Foram realizados ensaios de inflamabilidade horizontal (FMVSS 302) e vertical (ASTM D6413), utilizando gabinete metálico normalizado que suporta os dois métodos escolhidos.

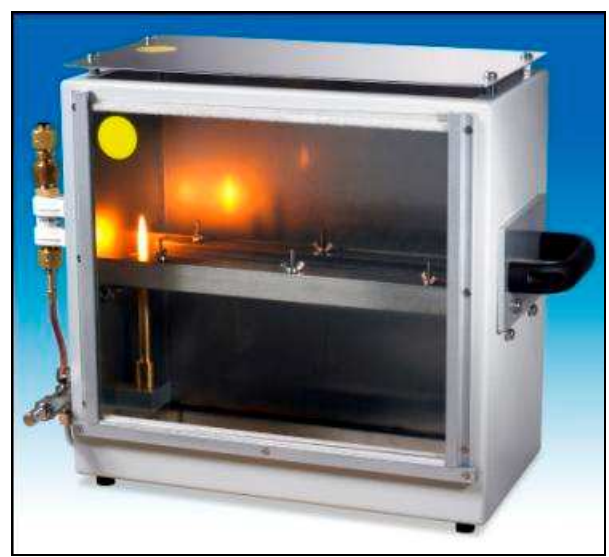

Figura 5: Gabinete normalizado

Fonte: http://www.fire-testing.com/whitedragon/documents/image/FMVSS\%20web.jpg.

\section{RESULTADOS}

\subsection{Ensaios iniciais.}

Ensaios realizados com as primeiras seis separações de misturas, Indicação (PES/C). 


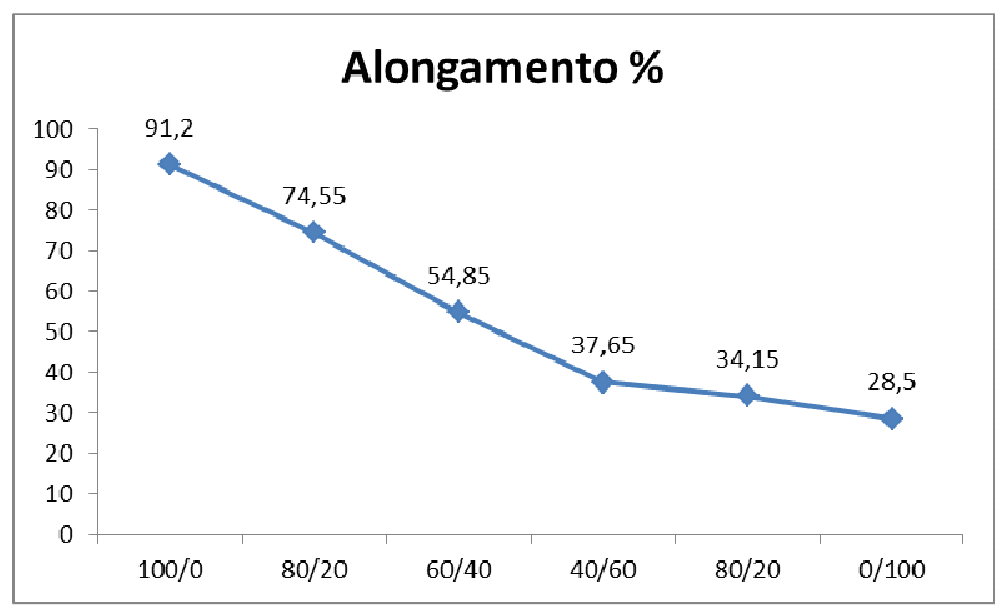

Gráfico 1: Alongamento \%.

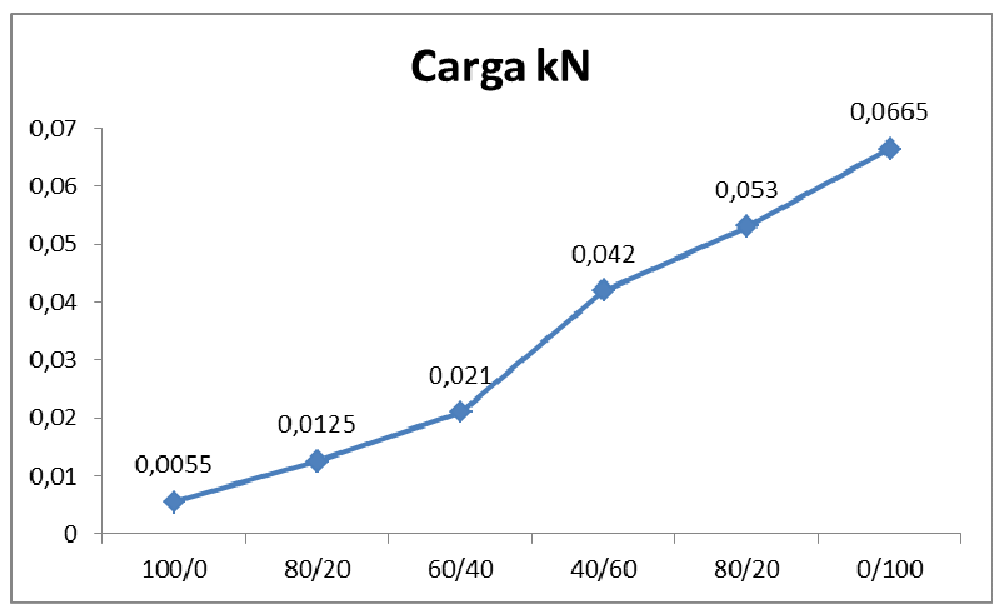

Gráfico 2: Carga de ruptura kN.

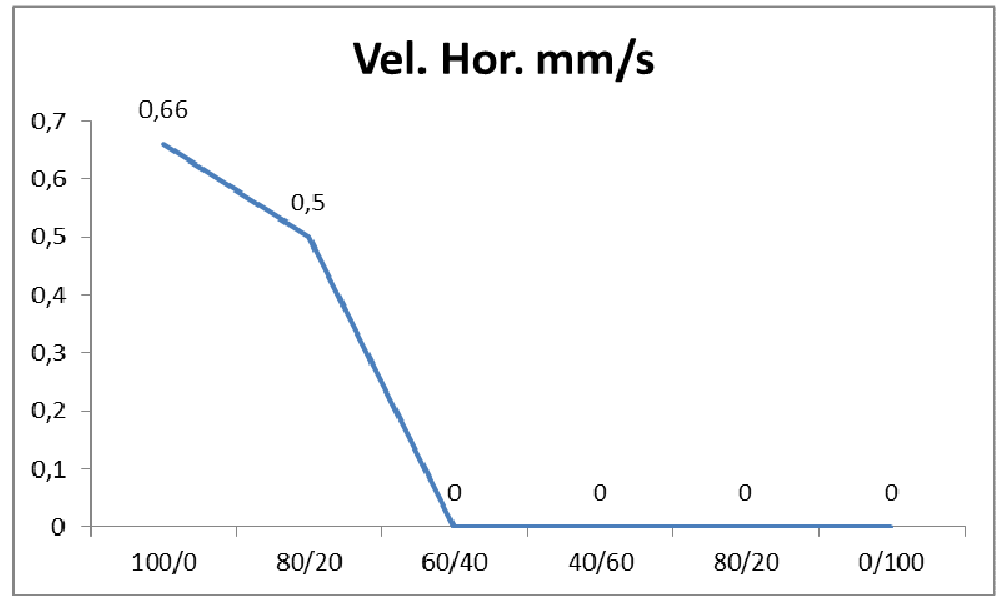

Gráfico 3: Velocidade de queima horizontal $\mathrm{mm} / \mathrm{s}$. 


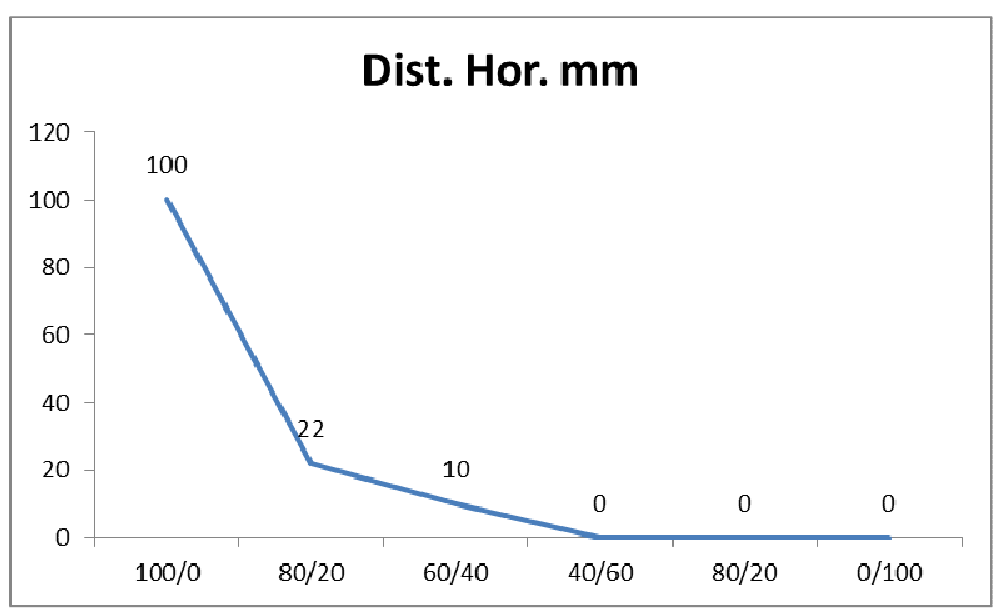

Gráfico 4: Distância queimada horizontal mm.

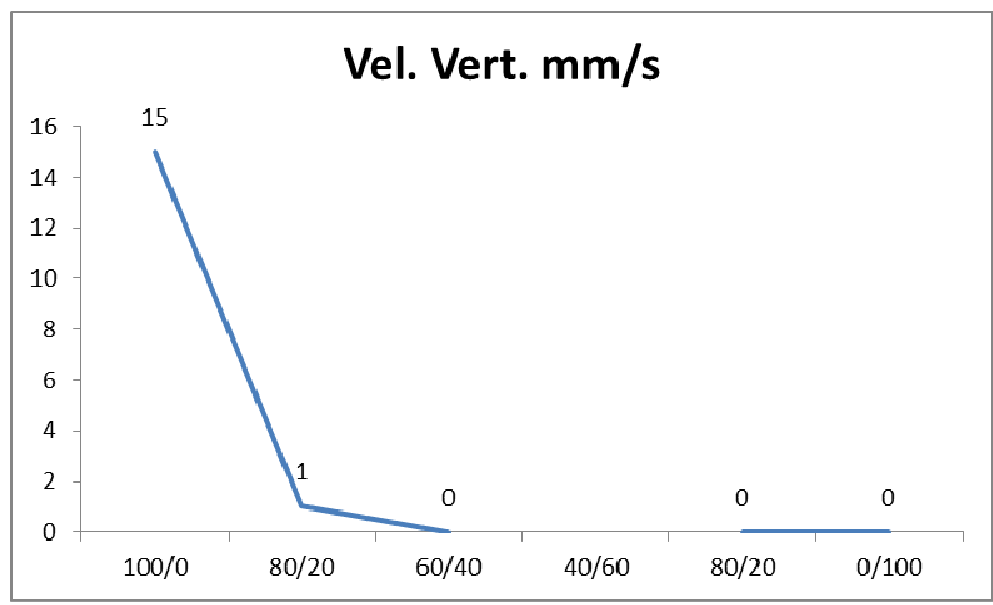

Gráfico 5: Velocidade de queima vertical mm/s.

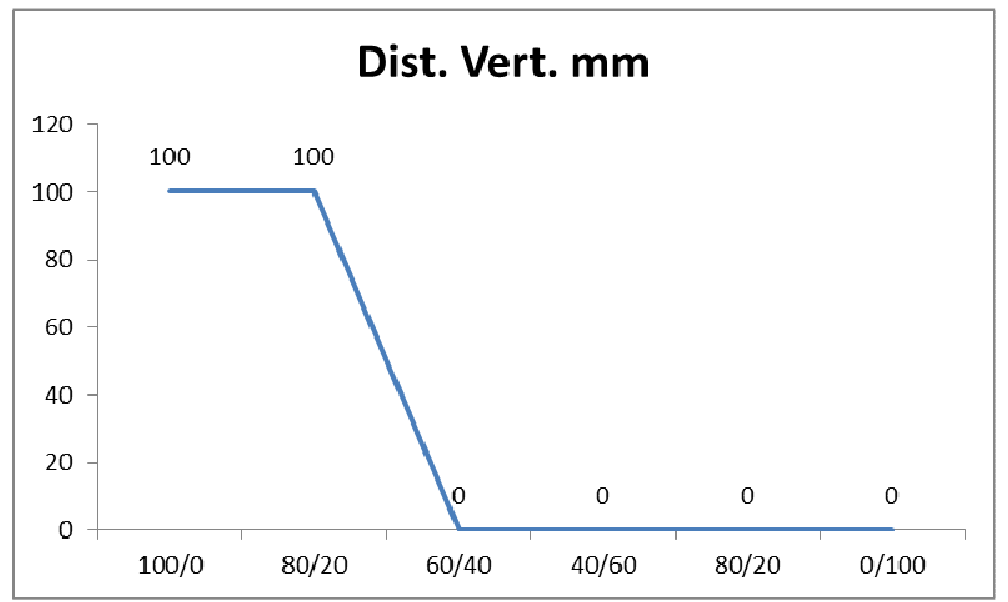

Gráfico 6: Distância queimada vertical mm.

3.2 Ensaios secundários.

Ensaios realizados com três amostras entre 100/0 e 80/20, Indicação (PES/C). 


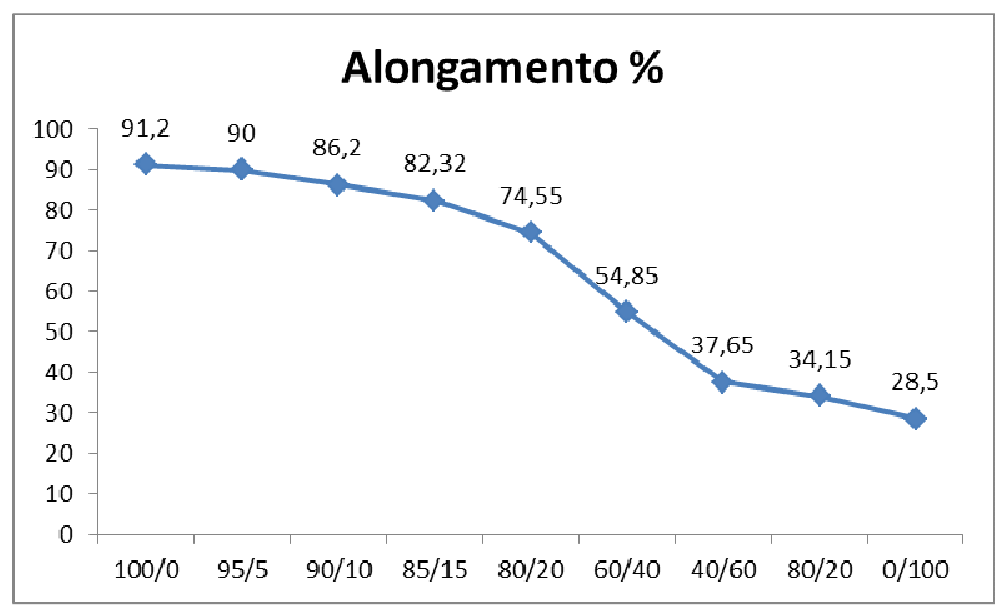

Gráfico 7: Alongamento \%.

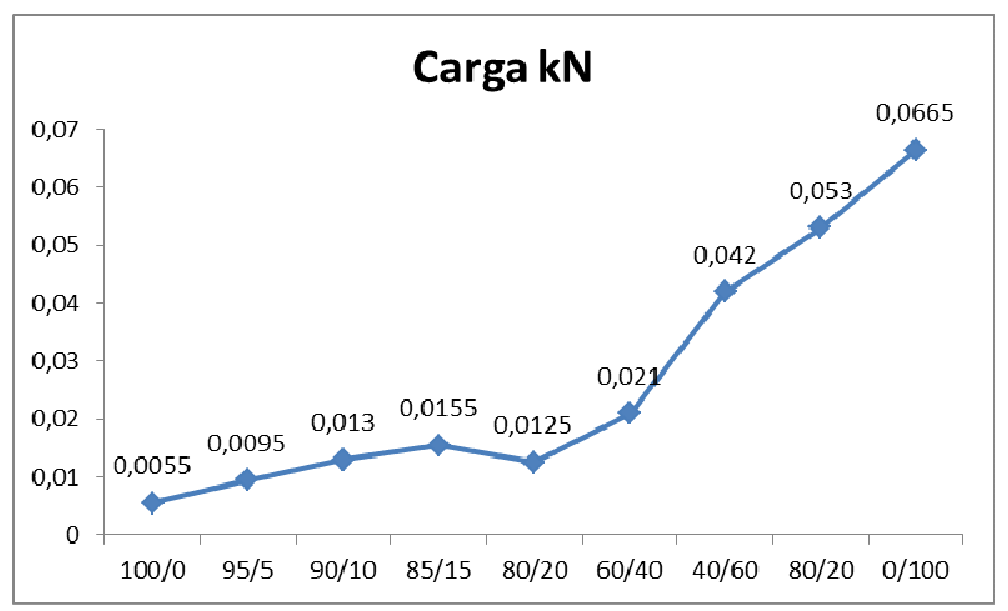

Gráfico 8: Carga de ruptura kN.

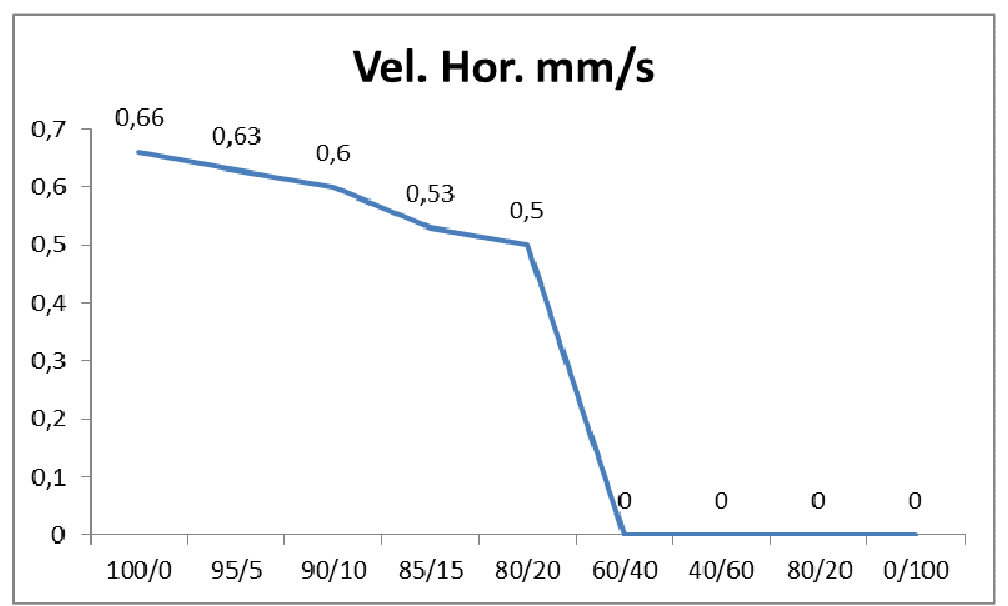

Gráfico 9: Velocidade de queima horizontal $\mathrm{mm} / \mathrm{s}$. 


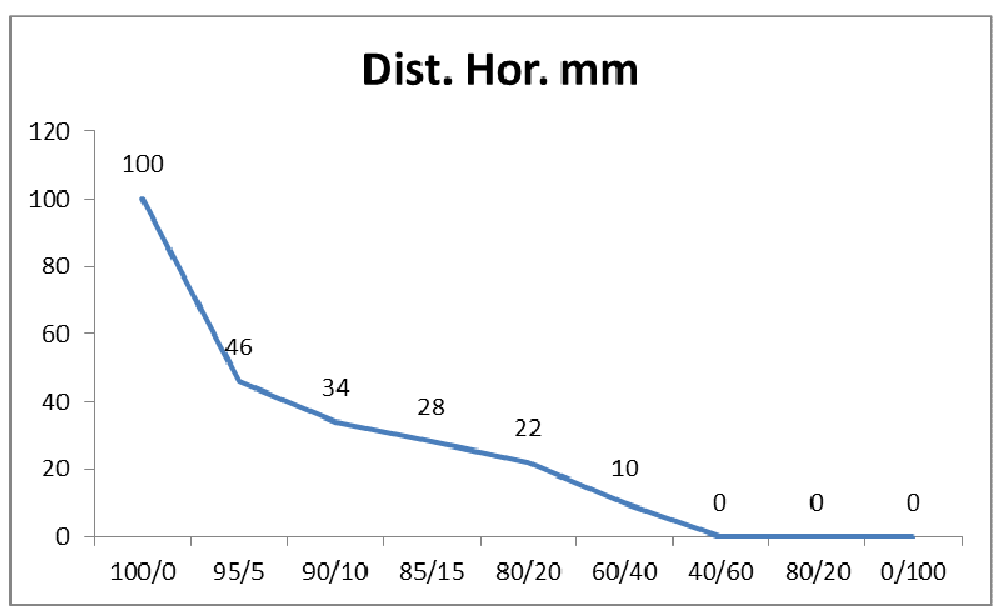

Gráfico 10: Distância queimada horizontal mm.

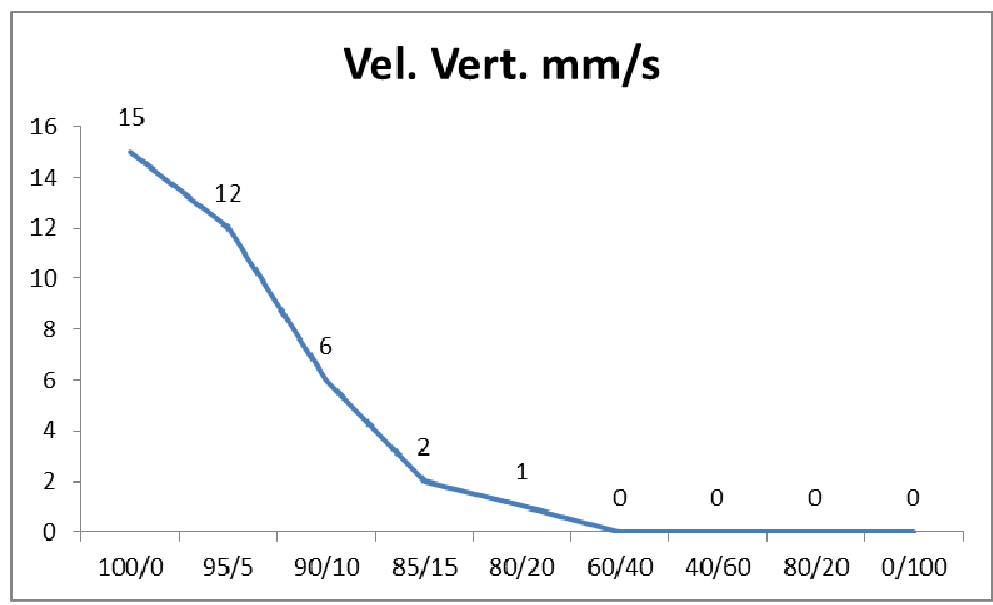

Gráfico 11: Velocidade de queima vertical $\mathrm{mm} / \mathrm{s}$.

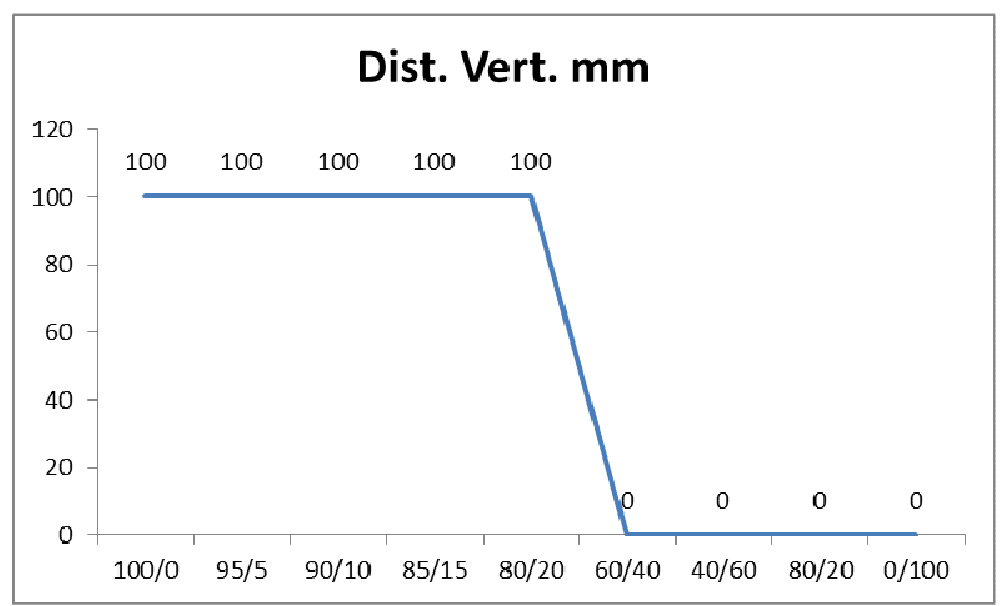

Gráfico 6: Distância queimada vertical mm.

\section{CONCLUSÃO}

O uso de fibras de carbono na composição de misturas íntimas juntamente com poliéster, para a produção de nãotecidos, obtiveram resultados ótimos em formulações a partir de $20 \%$ de 
carbono e bons resultados retardantes nas formulações $5 \%, 10 \%$ e $15 \%$, promovendo o retardo à chama.

Seu custo de produção pode ser beneficiado em comparação aos materiais atuais disponíveis no mercado, que utilizam tratamentos químicos e impregnações para alcançar o mesmo efeito retardante, uma vez que a mistura intima de fibras é realizada na linha de abertura sem a necessidade de processos e produtos adicionais.

De uma forma geral, mesmo que seja possível e viável a utilização da composição de $100 \%$ carbono, a mesma não pode ser fundida em um processo de termofixação, sendo necessário o uso de aglomerantes químicos ou outra fibra termoplástica.

Foi observado que devido à resistência a altas temperaturas, o comportamento da fibra de carbono não se altera de acordo com o tempo de exposição às chamas. Desta forma o material mantém sua capacidade retardante mesmo exposto a repetidas exposições.

\section{REFERÊNCIAS}

[1] FLOYD, K. L; TAYLOR, H. M. Industrial Applications of Textiles. Manchester: The Textile Institute, 1974.

[2] SCHWARTZ, M. M. Composite materials handbook. New York, St. Louis: Access Intelligence, c1984.

[3] HASSAN, Mohamed Behery; ALI, Demir. Synthetic Filament Yarn Texturing Technology. New Jersey: Prentice Hall, 1997.

[4] RUSSELL, S. J. Handbook of Nonwovens. Boca Raton, USA: The Textile Institute, 2007.

[5] PARIKH, D. V; SACHINVALA, N. D; CHEN, Y; SUN, L; BHAT, G; RAMKUMAR, S. Acoustic Properties of Environmentally Benign Automotive Natural Fiber Composites.

AATCC Review, January, 43-48, 2006.

[6] FUNG, Walter; HARDCASTLE, Mike. Textiles in Automitive Engineering.

Pennsylvania, USA: Woodhead Publishing, 2001.

[7] SCOTT, Richard A. Textiles For Protection. Cambridge, UK: Woodhead Publishing, 2005.

[8] ZUBKOVA, N. S; KARELINA, I. M; ZAITSEV, A.A; MERKULOV, A. A;

KONSTANTINOVA, N. I. Toxity of Combustion Products and Smoking of Decorative Finishing Fabrics. Fibre Chemistry, Vol 39 N 3, 2007.

FREEDY, Gustavo Rewald. Tecnologia dos Nãotecidos. São Paulo, 2006.

KRASNY, John F; PARKER, William J; BABRAUSKAS, Vytenis. Fire Behavior of Upholstered Furniture and Mattresses. New York, USA: Noyes Publications, 2000. GIBSON, Phillip W; LEE, Calvin; KO, Frank; RENEKER, Darrell. Application of Nanofiber Technology to Nonwoven Thermal Insulation. Journal of Engineered Fibers and Fabrics. USA, Volume 2, Issue 2, 2007.

TSUYUMOTO, Isao; MIURA, Yuichiro; HORI, Yukari. Fire-resistant nonwovens of EVOH and PET treated with amorphous sodium polyborate. J Mater Sci. Japan, 45 :2504-2509, 2010.

AMERICAN SOCIETY FOR TESTING MATERIALS. . ASTM D1230: 2010, Standard Test Method for Flammability of Apparel Textiles. 
ASTM D6413: 2011, Standard Test Method for Flame Resistance of Textiles

(Vertical Test)

FEDERAL MOTOR VEHICLE SAFETY STANDRAD

FMVSS 302: 2008, Flammability of materials used in the occupant compartments

of motor vehicles.

ASSOCIAÇÃO BRASILEIRA DE NORMAS TÉCNICAS.

.NBR 13041: 2004, Nãotecido - Determinação da resistência à tração e alongamento

- Método de tiras e "Grab". 